\title{
Transition systems over games
}

\author{
Paul Blain Levy \\ University of Birmingham \\ p.b.levy@cs.bham.ac.uk
}

\author{
Sam Staton \\ Radboud University Nijmegen \\ s.staton@cs.ru.nl
}

\begin{abstract}
We describe a framework for game semantics combining operational and denotational accounts. A game is a bipartite graph of "passive" and "active" positions, or a categorical variant with morphisms between positions.

The operational part of the framework is given by a labelled transition system in which each state sits in a particular position of the game. From a state in a passive position, transitions are labelled with a valid O-move from that position, and take us to a state in the updated position. Transitions from states in an active position are likewise labelled with a valid P-move, but silent transitions are allowed, which must take us to a state in the same position.

The denotational part is given by a "transfer" from one game to another, a kind of program that converts moves between the two games, giving an operation on strategies. The agreement between the two parts is given by a relation called a "stepped bisimulation".

The framework is illustrated by an example of substitution within a lambda-calculus.
\end{abstract}

Categories and Subject Descriptors F.3.2 [Logic and meanings of programs]: Semantics of programming languages

\section{Introduction}

This paper is concerned with two established lines of research in the semantics of higher-order calculi. One is game semantics using pointers [10], a form of denotational semantics that has been widely adapted successfully adapted to many language features, including general references [3] 27], control operators [19], exceptions [20] and polymorphism [22, 23]. The other is open (aka normal form) bisimulation [28], a convenient operational technique for establishing observational equivalences in various settings [13, 24, 25, 26, 29], based on a transition system constructed from the syntax of the calculus.

It is widely accepted that these two ideas have a lot in common, and that both operational and denotational perspectives are important [7, 14, 15, 16, 21]. The contribution of this paper is to elaborate the connections and to develop some principles that underlie the relationship.

\section{Summary of the concepts}

Our analysis is based on the following five key concepts:

Permission to make digital or hard copies of all or part of this work for personal or classroom use is granted without fee provided that copies are not made or distributed for profit or commercial advantage and that copies bear this notice and the full citation on the first page. Copyrights for components of this work owned by others than ACM must be honored. Abstracting with credit is permitted. To copy otherwise, or republish, to post on servers or to redistribute to lists, requires prior specific permission and/or a fee. Request permissions from permissions@acm.org.

CSL-LICS 2014, July 14-18, 2014, Vienna, Austria.

Copyright (C) 2014 ACM 978-1-4503-2886-9...\$15.00.

http://dx.doi.org/10.1145/2603088.2603150
1. A game, which is essentially a bipartite graph describing the moves that can be made as play passes between the different positions of the two players.

2. A strategy for a game, which specifies a particular way of playing a game, from a given starting position. Formally, it is a set of paths through the graph.

3. A transition system over a game, which is the operational part of the account. This can be thought of as an abstract machine that performs a strategy. Formally, it is a transition system labelled by legal moves of a game. (The set of legitimate actions changes over time, by contrast with conventional LTSs.)

4. A way to transfer strategies from one game to another, which is the denotational part of the account. We take as an example a composition operation, which takes two strategies in a particular game $\mathcal{G}$ and composes them. We understand this as a transfer from the tensor game $\mathcal{G} \otimes \mathcal{G}$ (whose strategies are roughly pairs of strategies) to the game $\mathcal{G}$.

5. A stepped bisimulation across a transfer, which relates the operational and denotational accounts. Our main theorem says that, if $x$ and $y$ are states of transition systems for $\mathcal{G}$ and $\mathcal{H}$ respectively, and they are stepped bisimilar across a transfer $\mathcal{O}$ : $\mathcal{G} \rightarrow \mathcal{H}$, then the strategy performed by $x$ is transfered by $\mathcal{O}$ to the strategy performed by $y$. For the example of composing strategies, we are able to relate the denotational operation of composition and the operational results of substitution.

\section{Goals and further discoveries}

When we embarked on this project, we had a number of goals. Primarily, we wanted to understand techniques for operating on strategies. We also wanted to set the fundamental notions within well-established mathematical frameworks, for example, transition systems as coalgebras, and renaming in terms of functor categories. As we report here, we have accomplished these things. But we have made some surprising discoveries too.

- We found a convenient diagrammatic notation for operations on strategies.

- Several well-known categorical concepts turn out to play an interesting role, in particular two-dimensional partial map (Sect. 5), bimodule (Sect. 8), and $*$-autonomous bicategory (Sect.9).

\section{Related Work}

Apart from papers about denotational and/or operational game semantics, we briefly comment on some work that might seem broadly related to our work. First, it seems appropriate to emphasise the difference between open bisimilarity, in which a function is tested by calling it with a fresh identifier, and applicative bisimilarity [1], in which a function is tested by calling it with all possible arguments. For the latter there remains a big gap between oper- 
ational and denotational accounts: Howe's ingenious congruence proof [9] appears unrelated to any denotational principles or models.

Second, we recall the 'bialgebraic semantics' of Turi and Plotkin [30], which relates operational and denotational semantics for simple first-order process calculi. But bialgebraic semantics seems different in spirit from our work. On the one hand bialgebraic semantics neatly explains when the structural operational semantics of a first-order process calculus is compositional by construction. On the other hand we are investigating compositionality properties for an abstract-machine-based operational semantics for higher-order calculi, where compositionality is not immediate.

In addition to these, there are many other operational analyses of game semantics, notably [5, 6] and the "traversal" technology of [4]. Games similar to those in Sect. 3]appear in [12, 17]. Bimodules (profunctors) are employed in game semantics in [8, 31].

\section{Illustrative Example}

\subsection{Example calculus}

The reader might expect at this point to see a rich example calculus, e.g. call-by-value typed $\lambda$-calculus with recursive types and general references. However, treating such a calculus would involve several complications (answer moves, ultimate pattern matching [25], renamings of references) that would distract somewhat from the points we are presenting. So instead we shall omit the general references, and consider a single recursive type

$$
A=(A \times A) \rightarrow 0
$$

which is just complicated enough to illustrate the points we are making in the paper. A value of this type is a function that is called with two arguments and (as in CPS) never returns. By abbreviating

$$
\lambda(\mathrm{x}, \mathrm{y}) \cdot M \stackrel{\text { def }}{=} \lambda \mathrm{z} . \mathrm{split} \mathrm{z} \text { as }(\mathrm{x}, \mathrm{y}) \cdot M
$$

we obtain the following untyped (or uni-typed) calculus. We stress that it has no intrinsic importance; it is merely an illustrative fragment of the rich calculus described above.

$$
\begin{array}{ll}
\text { Value } & V \quad::=\mathrm{x} \mid \lambda(\mathrm{x}, \mathrm{y}) \cdot M \\
\text { Nonreturning command } & M \quad:=V(V, V)
\end{array}
$$

where $\lambda$ binds $\mathrm{x}$ and y; we work up to $\alpha$-equivalence. For a finite set $\Gamma$ of identifiers, we write $\Gamma \vdash^{\vee} V$ to say that $V$ is a value with free identifiers drawn from $\Gamma$, and $\Gamma \vdash^{\text {nc }} M$ for a nonreturning command. We omit the typing rules, which are evident.

Operational semantics of an open command $\Gamma \vdash^{\mathrm{nc}} M$ is given by the $C$-machine, which $\beta$-reduces

$$
\Gamma \vdash^{\mathrm{nc}}(\lambda(\mathrm{x}, \mathrm{y}) \cdot M)(V, W) \rightsquigarrow M[V / \mathrm{x}, W / \mathrm{y}]
$$

until reaching a command of the form $\mathrm{x}(V, W)$ for some $\mathrm{x} \in \Gamma$.

\subsection{Example interaction}

We consider interaction between two players called $\mathrm{P}$ (Proponent, Patricia, the program) and $\mathrm{O}$ (Opponent, Oliver, the environment). Each passes functions to the other, and the passed functions are represented as fresh function-names. To illustrate this, consider the program:

$$
\mathrm{x}, \mathrm{y} \vdash^{\mathrm{nc}}(\lambda(\mathrm{s}, \mathrm{t}) \cdot \mathrm{s}(\mathrm{t}, \mathrm{y}))(\mathrm{x}, \lambda(\mathrm{p}, \mathrm{q}) \cdot(\lambda(\mathrm{u}, \mathrm{v}) \cdot \mathrm{u}(\mathrm{v}, \mathrm{q}))(\mathrm{p}, \mathrm{x}))
$$

For this program, here is an interaction between $\mathrm{P}$ and $\mathrm{O}$. Initially, $\mathrm{P}$ has function-names $\mathrm{x}, \mathrm{y}$ (i.e. has the ability to call them) and $\mathrm{O}$ has none.

1. P firstly performs one $\beta$-reduction, giving

$$
\mathrm{x}(\lambda(\mathrm{p}, \mathrm{q}) \cdot(\lambda(\mathrm{u}, \mathrm{v}) \cdot \mathrm{u}(\mathrm{v}, \mathrm{q}))(\mathrm{p}, \mathrm{x}), \mathrm{y})
$$

so she calls $\mathrm{x}$ and passes to $\mathrm{O}$ two function-names $b_{0}$ and $b_{1}$ representing the functions $\lambda(\mathrm{p}, \mathrm{q}) \cdot(\lambda(\mathrm{u}, \mathrm{v}) \cdot \mathrm{u}(\mathrm{v}, \mathrm{q}))(\mathrm{p}, \mathrm{x})$ and $\mathrm{y}$ respectively. These names are fresh, i.e. not used previously.

2. Suppose $\mathrm{O}$ calls $b_{0}$, passing to $\mathrm{P}$ two fresh function-names $\mathrm{w}_{0}$ and $\mathrm{w}_{1}$.

3. Then $\mathrm{P}$ executes $(\lambda(\mathrm{p}, \mathrm{q}) \cdot(\lambda(\mathrm{u}, \mathrm{v}) \cdot \mathrm{u}(\mathrm{v}, \mathrm{q}))(\mathrm{p}, \mathrm{x}))\left(\mathrm{w}_{0}, \mathrm{w}_{1}\right) \rightsquigarrow$ $\left(\lambda(\mathrm{u}, \mathrm{v}) \cdot \mathrm{u}\left(\mathrm{v}, \mathrm{w}_{1}\right)\right)\left(\mathrm{w}_{0}, \mathrm{x}\right) \rightsquigarrow \mathrm{w}_{0}\left(\mathrm{x}, \mathrm{w}_{1}\right)$ so she calls $\mathrm{w}_{0}$, passing two fresh function-names $b_{2}$ and $b_{3}$ representing the functions $\mathrm{x}$ and $\mathrm{w}_{1}$ respectively.

4. Suppose $\mathrm{O}$ calls $b_{1}$, passing to $\mathrm{P}$ two fresh function-names $\mathrm{w}_{2}$ and $\mathrm{w}_{3}$.

5. Then $P$ executes $y\left(w_{2}, w_{3}\right)$, i.e. immediately calls $y$, passing to $\mathrm{O}$ two fresh function-names $b_{4}$ and $b_{5}$ representing the functions $\mathrm{w}_{2}$ and $\mathrm{w}_{3}$ respectively.

We see that each player moves by calling a function-name from their inventory, which grows over time. Consequently the set of legitimate moves keeps changing.

Remark We are not explicitly using justification pointers in the style of [10] but it is clear that, for example, when $O$ calls $b_{1}$ in move 4 , he could express this as "calling the second name I received in move 1". That is: as a justification pointer with some extra data. On the other hand, when $\mathrm{P}$ calls y in move 5, there is no justification pointer because she owned y at the start.

\section{Games, Strategies, Transition Systems}

We consider two kinds of games in this paper: a familiar "discrete" kind in this section, and a more sophisticated "categorical" kind in Sect.4

\subsection{Games}

DeFINITION 1. A (discrete) game $\mathcal{G}$ is a bipartite directed multigraph. Explicitly:

- a set $\mathcal{G}^{\text {pass }}$ of passive positions

- a set $\mathcal{G}^{\text {act }}$ of active positions

- for each passive position $P$,

- a set Omove $P$ of Opponent-moves from $P$

- for each $m \in$ Omove $P$ an active result position P.m

- for each active position $Q$,

- a set Pmove $Q$ of Proponent-moves from $Q$

- for each $n \in$ Pmove $Q$ a passive result position $Q . n$.

Note that no position is designated "initial". We write $P{ }^{\circ}{ }^{m}>Q$ to mean that $m \in$ Omove $P$ and $Q=P . m$, and we write $Q \stackrel{n}{\longrightarrow} P$ to mean that $n \in$ Pmove $Q$ and $P=Q . n$.

Our main example of a game uses the notion of a gen-set, intuitively a set of names with a facility for generating fresh ones. Formally it is a set $A$ equipped with a set of permitted finite subsets of $A$, and for each permitted set $R$ an element $\nu R \in A \backslash R$ such that $R^{+} \stackrel{\text { def }}{=} R \cup\{\nu R\}$ is permitted. Here are some examples.

1. $\mathbb{N}$ is a gen-set with $\$ n \stackrel{\text { def }}{=}\{m \in \mathbb{N} \mid m<n\}$ permitted for all $n \in \mathbb{N}$, and $\nu(\$ n)=n$. This is similar to O's function-names in our example, viz. $b_{0}, b_{1}, b_{2}, b_{3}, b_{4}, b_{5}$.

2. If $B$ is a set then $B+\mathbb{N}$ is a gen-set with $U+\$ n$ permitted for all finite $U \subseteq B$ and $n \in \mathbb{N}$, and $\nu(U+\$ n)=$ inr $n$. This is similar to P's function-names in our example, viz. $\mathrm{x}, \mathrm{y}, \mathrm{w}_{0}, \mathrm{w}_{1}, \mathrm{w}_{2}, \mathrm{w}_{3}$. We would take $B$ to be some set containing $\mathrm{x}$ and $\mathrm{y}$.

3. [Example with thanks to L. Moss; not used in the sequel.] The universe of sets forms a gen-set, or strictly speaking a gen-class, 
with every finite set $R$ permitted and $\nu R \stackrel{\text { def }}{=}\{x \in R \mid x \notin x\}$, which is simply $R$ if the Axiom of Foundation is assumed.

For a permitted set $R$ and $n \in \mathbb{N}$, we write $R^{+n} \stackrel{\text { def }}{=} \overbrace{R^{+\cdots+}}^{n}$ and $\nu_{n} R \stackrel{\text { def }}{=} \nu R^{+n}$, giving a sequence $\left(\nu_{n} R\right)_{n \in \mathbb{N}}$ of distinct names not in $R$. For permitted sets $R, S$ and function $f: R \rightarrow S$ and $n \in \mathbb{N}$, we write $f^{+n}: R^{+n} \rightarrow S^{+n}$ for the extended function $f\left[\nu_{i} R \mapsto \nu_{i} S\right]_{i<n}$.

Let us now fix two gen-sets of $P$ 's function-names and of $O$ 's function-names respectively. We form a game $\lambda$ Game in which

- a passive position is a pair $\Gamma \| \Delta$, where $\Gamma$ and $\Delta$ are permitted sets of P's names and O's names respectively

- an active position is the same

- an O-move from $\Gamma \| \Delta$ is $b \in \Delta$, with result position $\Gamma^{+2} \| \Delta$

- a P-move from $\Gamma \| \Delta$ is $\mathrm{x} \in \Gamma$, with result position $\Gamma \| \Delta^{+2}$.

This is in keeping with the interaction in Section 2 $\mathrm{O}$ moves by selecting a function-name from his inventory, putting two new function-names into P's, and vice versa.

\subsection{Strategies}

Let $\mathcal{G}$ be a game. A play in $\mathcal{G}$ is any sequence of consecutive moves. Strategies may be described as sets of plays, as follows.

DEFINITION 2. 1. A strategy for $\mathcal{G}$ starting from a passive position $P$ is a set $\sigma$ of passive-ending plays

$$
P \stackrel{m_{0}}{\longrightarrow} \cdot \stackrel{n_{0}}{\longrightarrow} . \quad \ldots . \quad \cdot \stackrel{m_{r-1}}{\longrightarrow} \cdot \stackrel{n_{r-1}}{\longrightarrow} .
$$

such that the empty play is in $\sigma$, and smn $\in \sigma$ implies $s \in \sigma$, and $t n, t n^{\prime} \in \sigma$ implies $n=n^{\prime}$. We write Strat $_{\mathcal{G}}^{\text {pass }} P$ for the set of all such strategies.

2. A strategy for $\mathcal{G}$ starting from an active position $Q$ is a set $\sigma$ of passive-ending plays

$$
Q \stackrel{n_{0}}{\longrightarrow} \cdot \stackrel{m_{0}}{\longrightarrow} \cdot \quad \cdots \quad . \bullet^{n_{r}} \longrightarrow .
$$

such that smn $\in \sigma$ implies $s \in \sigma$, and $t n, t n^{\prime} \in \sigma$ implies $n=n^{\prime}$. We write $\operatorname{Strat}_{\mathcal{G}}^{\text {act }} Q$ for the set of all such strategies.

\subsection{Transition systems}

In the following, it is essential not to confuse positions with states. For a computer program playing chess, the position describes the current arrangement of the chessboard, which determines what moves are legitimate, whereas a state describes the values of memory cells etc. used to determine how to play. Think of a position as the "type" of a state.

DEFINITION 3. A small-step system over a game $\mathcal{G}$ consists of the following data.

- For each passive position $P$ a set $\mathbb{S}^{\text {pass }} P$ of passive states in position $P$.

- For each active position $Q$ a set $\mathbb{S}^{\text {act }} Q$ of active states in position $Q$.

- For each passive position P a function

$$
\zeta_{P}^{\text {pass }}: \mathbb{S}^{\text {pass }} P \quad \rightarrow \quad \prod_{m \in \text { Omove } P} \mathbb{S}^{\text {act }} P . m
$$

- For each active position $Q$ a function

$$
\zeta_{Q}^{\text {act }}: \mathbb{S}^{\text {act }} Q \rightarrow\left(\sum_{n \in \text { Pmove } Q} \mathbb{S}^{\text {pass }} Q . n\right)+\mathbb{S}^{\text {act }} Q
$$

For $x \in \mathbb{S}^{\text {pass }} P$ and $m \in$ Omove $P$ we write $x @ m$ for $\zeta_{P}^{\text {pass }}(x)(m)$. For $y \in \mathbb{S}^{\text {act }} P$ we write $y \stackrel{n}{\rightsquigarrow} x$ or $y \rightsquigarrow z$ (silent transition) according as $\zeta_{P}^{\text {act }}(y)$ is inl $(n, x)$ or inr $z$.

For our main example (\$2), we form a transition system $\lambda$ Syst over $\lambda$ Game as follows.

- A passive state in (passive) position $\Gamma \| \Delta$ is a family of values $\left(V_{a}\right)_{a \in \Delta}$, where $\Gamma \vdash^{\vee} V_{a}$ for each $a \in \Delta$.

- An active state in (active) position $\Gamma \| \Delta$ consists of a family of values $\left(V_{a}\right)_{a \in \Delta}$ and a nonreturning command $M$, where $\Gamma \vdash^{v}$ $V_{a}$ for each $a \in \Delta$ and $\Gamma \vdash^{\mathrm{nc}} M$. We write $\left(V_{a}\right)_{a \in \Delta} \triangleright M$ for an active state.

- For a passive state $\left(V_{a}\right)_{a \in \Delta}$ in position $\Gamma \| \Delta$, we define $\left(V_{a}\right)_{a \in \Delta} @ a \stackrel{\text { def }}{=}\left(V_{a}\right)_{a \in \Delta} \triangleright V_{a}\left(\nu_{0} \Gamma, \nu_{1} \Gamma\right)$.

- For an active state $\left(V_{a}\right)_{a \in \Delta} \triangleright M$ in position $\Gamma \| \Delta$, we set

$$
\left(V_{a}\right)_{a \in \Delta} \triangleright M\left\{\begin{array}{lll}
\rightsquigarrow & \left(V_{a}\right)_{a \in \Delta} \triangleright M^{\prime} & \text { if } M \rightsquigarrow M^{\prime} \\
\stackrel{x}{\rightsquigarrow} & \left(V_{a}\right)_{a \in \Delta}\left[\nu_{i} \Delta \mapsto V_{i}\right]_{i=0,1} \\
& \text { if } M=\mathrm{x}\left(V_{0}, V_{1}\right)
\end{array}\right.
$$

We often want to ignore the silent transitions, in which case we use the following.

DEFINITION 4. A big-step system over a game $\mathcal{G}$ consists of the following data.

- For each passive position $P$ a set $\mathbb{S}^{\text {pass }} P$ of passive states in position $P$.

- For each active position $Q$ a set $\mathbb{S}^{\text {act }} Q$ of active states in position $Q$.

- For each passive position P a function

$$
\zeta_{P}^{\text {pass }}: \mathbb{S}^{\text {pass }} P \rightarrow \prod_{m \in \text { Omove } P} \mathbb{S}^{\text {act }} P . m
$$

- For each active position $Q$ a function

$$
\zeta_{Q}^{\text {act }}: \mathbb{S}^{\text {act }} Q \rightarrow\left(\sum_{n \in \operatorname{Pmove} Q} \mathbb{S}^{\text {pass }} Q . n\right)+1
$$

For $x \in \mathbb{S}^{\text {pass }} P$ and $m \in$ Omove $P$ we write $x @ m$ for $\zeta_{P}^{\text {pass }}(x)(m)$. For $y \in \mathbb{S}^{\text {act }} P$ we write $y \stackrel{n}{\Longrightarrow} x$ and $y \Uparrow$ according as $\zeta_{P}^{\text {act }}(y)$ is inl $(n, x)$ or inr (). A small-step system always gives rise to a big-step one: we set $y \stackrel{n}{\Longrightarrow} x$ when $y \rightsquigarrow^{*} \stackrel{n}{\rightsquigarrow} x$, and $y \Uparrow$ when $y \rightsquigarrow^{\omega}$.

We may go a step further and dispense with the active states:

DEFINITION 5. A passive system over a game $\mathcal{G}$ consists of the following data.

- For each passive position $P$ a set $\mathbb{S}^{\text {pass }} P$ of passive states in position $P$.

- For each passive position $P$ a function $\zeta_{P}^{\text {pass }}: \mathbb{S}^{\text {pass }} P \rightarrow$

$$
\prod_{m \in \text { Omove } P}\left(\left(\sum_{n \in \text { Pmove } P . m} \mathbb{S}^{\text {pass }} \text { P.m.n }\right)+1\right)
$$

For $x \in \mathbb{S}^{\text {pass }} P$ and $m \in$ Omove $P$ we write $x @ m \stackrel{n}{\rightsquigarrow} w$ and $x @ m \Uparrow$ according as $\zeta_{P}^{\text {act }}(x)(m)$ is inl $(n, w)$ or inr () . (Here $@ \rightsquigarrow$ and @介 are quaternary and binary predicates respectively, and $x @ m$ has no meaning in a passive system.) Clearly a big-step system gives rise to a passive one by taking just the passive states.

We could consider bisimulations for small-step or big-step systems, but it turns out that the most useful notion is for passive systems.

DEFINITION 6. Let $\mathbb{S}$ be a passive system over a game $\mathcal{G}$. A passive bisimulation on $\mathbb{S}$ associates to each passive position $P$ a binary relation $\mathcal{R}_{P}$ on $\mathbb{S}^{\text {pass }} P$, such that if $x\left(\mathcal{R}_{P}\right) x^{\prime}$ and $m \in$ Omove $P$, either 
- $x @ m \stackrel{n}{\Longrightarrow} w$ and $x^{\prime} @ m \stackrel{n}{\Longrightarrow} w^{\prime}$ for some $n \in$ Pmove P.m and $w\left(\mathcal{R}_{P . m . n}\right) w^{\prime}$

- or $x @ m \Uparrow$ and $x^{\prime} @ m$ 介.

\subsection{From transition systems to strategies}

Each state has an associated strategy that describes the plays it may perform.

Proposition 1. 1. Let $\mathbb{S}$ be a passive system over a game $\mathcal{G}$, and $x$ a passive state in position $P$. Write $\llbracket x \rrbracket_{P}^{\text {pass }}$ for the set of traces of $x$, i.e. passive-ending plays $m_{0} n_{0} \ldots m_{k-1} n_{k-1}$ from $P$ that arise from a sequence of states

$$
x=x_{0} \quad x_{0} @ m_{0} \stackrel{n_{0}}{\rightsquigarrow} x_{1} \quad \cdots \quad x_{k-1} @ m_{k-1}{\stackrel{n_{k-1}}{\rightsquigarrow}} x_{k}
$$

Then $\llbracket x \rrbracket_{P}^{\text {pass }} \in \operatorname{Strat}_{\mathcal{G}}^{\text {pass }} P$.

2. Let $\mathbb{S}$ be a big-step system over a game $\mathcal{G}$, and y an active state in position $Q$. Write $\llbracket y \rrbracket_{Q}^{\text {act }}$ for the set of traces of $y$ i.e. passiveending plays $n_{0} m_{0} n_{1} \ldots m_{k-1} n_{k}$ from $Q$ that arise from a sequence of states

$$
y \stackrel{n_{0}}{\rightsquigarrow} x_{0} \quad x_{0} @ m_{0} \stackrel{n_{1}}{\rightsquigarrow} x_{1} \quad \cdots \quad x_{k-1} @ m_{k-1} \stackrel{n_{k}}{\rightsquigarrow} x_{k}
$$

Then $\llbracket y \rrbracket_{Q}^{\text {act }} \in \operatorname{Strat}_{\mathcal{G}}^{\text {act }} Q$.

As usual for deterministic systems, trace equivalence and bisimilarity coincide:

Proposition 2. 1. Let $\mathbb{S}$ be a passive system over a game $\mathcal{G}$, and $x, x^{\prime}$ passive states in position $P$. Then $\llbracket x \rrbracket_{P}^{\text {pass }}=\llbracket x^{\prime} \rrbracket_{P}^{\text {pass }}$ iff there is a passive bisimulation $\mathcal{R}$ on $\mathbb{S}$ such that $x\left(\mathcal{R}_{P}\right) x^{\prime}$.

2. Let $\mathbb{S}$ be a big-step system over a game $\mathcal{G}$, and $y, y^{\prime}$ active states in position $Q$. Then $\llbracket y \rrbracket_{Q}^{\text {act }}=\llbracket y^{\prime} \rrbracket_{Q}^{\text {pass }}$ iff there is a passive bisimulation $\mathcal{R}$ on $\mathbb{S}$ such that either

- $y \stackrel{n}{\Longrightarrow} w$ and $y^{\prime} \stackrel{n}{\Longrightarrow} w^{\prime}$ for some $n \in$ Pmove P.m and $w\left(\mathcal{R}_{Q . n}\right) w^{\prime}$

- or $y \Uparrow$ and $y^{\prime} \Uparrow$.

\section{Position Morphisms}

We return to our example (2). Given functions $p: \Gamma \rightarrow \Gamma^{\prime}$ and $q: \Delta^{\prime} \rightarrow \Delta$, any passive state $\left(V_{a}\right)_{a \in \Delta}$ in position $\Gamma \| \Delta$ can be transformed into a passive state $\left(p^{*} V_{q(a)}\right)_{a \in \Delta^{\prime}}$ in position $\Gamma^{\prime} \| \Delta^{\prime}$, where $p^{*}$ indicates renaming. We would expect that the operational meaning of the latter state can be obtained from that of the former by the following operation transforming strategies $\sigma$ on $\Gamma \| \Delta$ to strategies on $\Gamma^{\prime} \| \Delta^{\prime}$.

An O-move from the latter position is converted into an $O$-move from the former by applying $q$. If we feed this to $\sigma$ and it responds with a $P$-move from $\Gamma^{+2} \| \Delta$, we play a P-move from $\Gamma^{\prime+2} \| \Delta^{\prime}$ by applying $p^{+2}$. If we receive another O-move, we continue in the same way.

The correctness of this construction is an instance of a general fact, Proposition 5 below. The key idea is that the pair $p \| q$ in the preceding discussion may be called a morphism $\Gamma\left\|\Delta \rightarrow \Gamma^{\prime}\right\| \Delta^{\prime}$, and it is then evident that the passive positions form a category (and likewise the active positions).

\subsection{Categorical games}

DEFInITION 7. A categorical game $\mathcal{G}$ is a game together with the following additional data.

- For each pair of passive positions $P, P^{\prime}$, a set $\mathcal{G}^{\text {pass }}\left(P, P^{\prime}\right)$ of passive position morphisms $P \rightarrow P^{\prime}$.

- For two passive position morphisms $P \stackrel{f}{\longrightarrow} P^{\prime} \stackrel{f^{\prime}}{\longrightarrow} P^{\prime \prime}$ a composite $P \stackrel{f ; f^{\prime}}{\longrightarrow} P^{\prime \prime}$, and an identity $P \stackrel{\operatorname{id}_{P}}{\longrightarrow} P$ for each passive position $P$, satisfying the usual left and right identity and associativity laws.

- Likewise for active positions.

- For each passive position morphism $f: P \rightarrow P^{\prime}$ and $m^{\prime} \in$ Omove $P^{\prime}$, a move (Omove $\left.f\right)\left(m^{\prime}\right) \in$ Omove $P$ and active position morphism $f . m^{\prime}: P$.(Omove $\left.f\right)\left(m^{\prime}\right) \rightarrow P^{\prime} . m^{\prime}$, satisfying equations for identity and composition described below.

- For each active position morphism $g: Q \rightarrow Q^{\prime}$ and each $n \in$ Pmove $Q$, a move (Pmove $g)(n) \in$ Pmove $Q^{\prime}$ and passive position morphism g.n : Q.n $\rightarrow Q^{\prime}$.(Pmove $g$ ) $(n)$, satisfying equations for identity and composition described below.

We introduce a helpful diagrammatic notation. We shall draw an $O$-move square

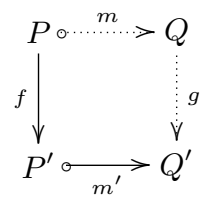

to say that $m=($ Omove $f)\left(m^{\prime}\right)$ and $g=f . m^{\prime}$, and likewise draw a $P$-move square

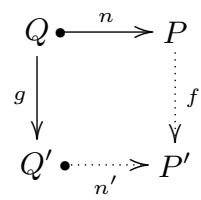

to say that $n^{\prime}=($ Pmove $g)(n)$ and $f=g . n$. Note the conventions used: downwards arrows are position morphisms, rightwards arrows are moves, and dashed arrows are derived.

The equations mentioned in Def 7 stipulate that identities and composites of O-move squares
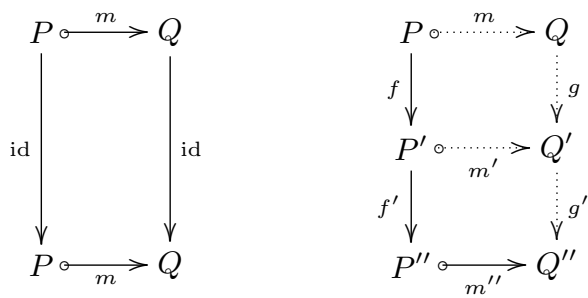

are O-move squares, and likewise for P-move squares.

We may now describe how our main example $\lambda$ Game forms a categorical game. In both the passive and active position categories a morphism

$$
p\|q: \Gamma\| \Delta \rightarrow \Gamma^{\prime} \| \Delta^{\prime}
$$

consists of functions $p: \Gamma \rightarrow \Gamma^{\prime}$ and $q: \Delta^{\prime} \rightarrow \Delta$, with identity and composite morphisms defined in the evident way. The squares are

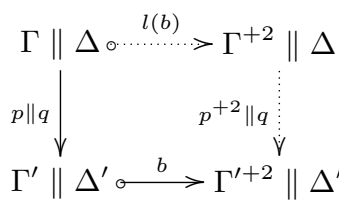

O-move square

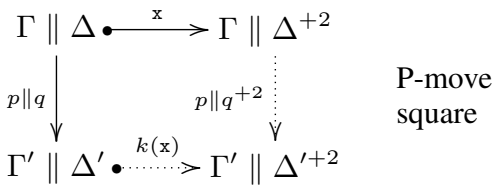




\subsection{Position morphisms acting on strategies}

The operation on strategies described at the start of the section may now be given generally. Let $\mathcal{G}$ be a categorical game. A $\mathcal{G}$ interaction sequence is just a sequence of O-move and $\mathrm{P}$-move squares, e.g.

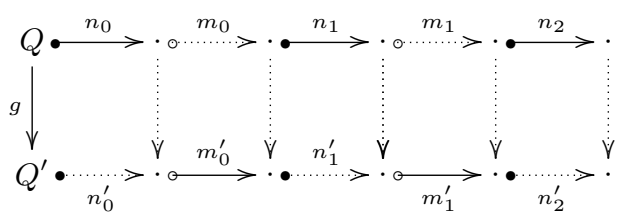

We may describe this interaction sequence from $g$ by the sequence of moves $n_{0} m_{0}^{\prime} n_{1} m_{1}^{\prime} n_{2}$ depicted as solid, since they determine the other moves and morphisms. The play depicted along an interaction sequence's upper edge is its internal play; the one depicted along its lower edge is its external play.

Proposition 3. For any passive position morphism $f: P \rightarrow P^{\prime}$ and $\sigma \in \operatorname{Strat}_{\mathcal{G}}^{\text {pass }} P$, let $\left(\operatorname{Strat}_{\mathcal{G}}^{\text {pass }} f\right)(\sigma)$ be the set of external plays of passive-ending $\mathcal{G}$-interaction sequences from $f$ whose internal play is in $\sigma$. Then $\left(\operatorname{Strat}_{\mathcal{G}}^{\text {pass }} f\right)(\sigma) \in \operatorname{Strat}_{\mathcal{G}}^{\text {pass }} P^{\prime}$. Likewise for an active position morphism.

Proposition 4. (Functoriality of Strat $_{\mathcal{G}}^{\text {pass }}$ and Strat $_{\mathcal{G}}^{\text {act }}$ )

1. For a strategy $\sigma \in \operatorname{Strat}_{\mathcal{G}}^{\text {pass }} P$ we have

$$
\left(\text { Strat }_{\mathcal{G}}^{\text {pass }} \operatorname{id}_{P}\right)(\sigma)=\sigma
$$

and likewise for active positions.

2. For passive position morphisms $P \stackrel{f}{\longrightarrow} P^{\prime} \stackrel{g}{\longrightarrow} P^{\prime \prime}$, and strategy $\sigma \in \operatorname{Strat}_{\mathcal{G}}^{\text {pass }} P$, we have

$$
\left(\operatorname{Strat}_{\mathcal{G}}^{\text {pass }}(f ; g)\right)(\sigma)=\left(\operatorname{Strat}_{\mathcal{G}}^{\text {pass }} g\right)\left(\operatorname{Strat}_{\mathcal{G}}^{\text {pass }} f\right)(\sigma)
$$

and likewise for active position morphisms.

\subsection{Transition systems over a categorical game}

As in Sect 3.3 we define small-step, big-step and passive systems over a categorical game.

DEFINITION 8. Let $\mathcal{G}$ be a categorical game. A small-step system over $\mathcal{G}$ is a small-step system over the discrete game $\mathcal{G}$ (as in Definition 3), with the following additional data.

- For each passive position morphism $f: P \rightarrow P^{\prime}$ a function $\mathbb{S}^{\text {pass }} f: \mathbb{S}^{\text {pass }} P \rightarrow \mathbb{S}^{\text {pass }} P^{\prime}$.

- For each active position morphism $g: Q \rightarrow Q^{\prime}$ a function $\mathbb{S}^{\text {act }} g: \mathbb{S}^{\text {act }} Q \rightarrow \mathbb{S}^{\text {act }} Q^{\prime}$.

The following conditions must be satisfied.

- For $x \in \mathbb{S}^{\text {pass }} P$ we have $\left(\mathbb{S}^{\text {pass }} \operatorname{id}_{P}\right)(x)=x$, and likewise for active positions.

- For passive position morphisms $P \stackrel{f}{\longrightarrow} P^{\prime} \stackrel{g}{\longrightarrow} P^{\prime \prime}$ and $x \in \mathbb{S}^{\text {pass }} P$ we have $\left(\mathbb{S}^{\text {pass }} f ; g\right)(x)=\left(\mathbb{S}^{\text {pass }} g\right)\left(\mathbb{S}^{\text {pass }} f\right)(x)$, and likewise for active position morphisms.

- For every O-move square (1) and $x \in \mathbb{S}^{\text {pass }} P$, we have $\left(\mathbb{S}^{\text {act }} g\right)(x @ m)=\left(\left(\mathbb{S}^{\text {pass }} f\right)(x)\right) @ m^{\prime}$.

- For every active position morphism $g: Q \rightarrow Q^{\prime}$ and $y \in$ $\mathbb{S}^{\text {act }} Q$, if $y \rightsquigarrow z$ then $\left(\mathbb{S}^{\text {act }} g\right)(y) \rightsquigarrow\left(\mathbb{S}^{\text {act }} g\right)(z)$.

- For every P-move square (2) and $y \in \mathbb{S}^{\text {act }} Q$, if $y \stackrel{n}{\rightsquigarrow} x$ then $\left(\mathbb{S}^{\text {act }} g\right)(y) \stackrel{n^{\prime}}{\rightsquigarrow}\left(\mathbb{S}^{\text {pass }} f\right)(x)$.

We likewise define a big-step system and passive system over $\mathcal{G}$. Once again a small-step system gives rise to a big-step system, and a big-step system to a passive system.
Our example $\lambda$ Syst forms a small-step system over the categorical game $\lambda$ Game: a passive morphism (3) transforms $\left(V_{a}\right)_{a \in \Delta}$ to $\left(p^{*} V_{q(b)}\right)_{b \in \Delta^{\prime}}$, and an active morphism (3) transforms $\left(V_{a}\right)_{a \in \Delta}$ $M$ to $\left(p^{*} V_{q(b)}\right)_{b \in \Delta^{\prime}} \triangleright p^{*} M$.

\subsection{Compositionality theorem for position morphisms}

We now substantiate the claim at the start of the section: it is an instance of the following "compositionality theorem".

PROPOSITION 5. (Naturality of $\llbracket-\rrbracket^{\text {pass }}$ and $\llbracket-\rrbracket^{\text {act }}$ )

1. Let $\mathbb{S}$ be a passive system over a categorical game $\mathcal{G}$. For any passive position morphism $f: P \rightarrow P^{\prime}$ and state $x \in \mathbb{S}^{\text {pass }} P$ we have

$$
\llbracket\left(\mathbb{S}^{\text {pass }} f\right)(x) \rrbracket_{P^{\prime}}^{\text {pass }}=\left(\text { Strat }_{\mathcal{G}}^{\text {pass }} f\right) \llbracket x \rrbracket_{P}^{\text {pass }}
$$

2. Let $\mathbb{S}$ be a big-step system over a categorical game $\mathcal{G}$. For any active position morphism $g: Q \rightarrow Q^{\prime}$ and state $y \in \mathbb{S}^{\text {act }} Q$ we have

$$
\llbracket\left(\mathbb{S}^{\text {act }} g\right)(y) \rrbracket_{Q^{\prime}}^{\text {act }}=\left(\operatorname{Strat}_{\mathcal{G}}^{\text {act }} g\right) \llbracket y \rrbracket_{Q}^{\text {act }}
$$

\section{Categorical Games via Two-Dimensional Partial Maps}

In this section, which is not used in the sequel, we present a concise formulation of categorical game in terms of two-dimensional partial maps [18].

DEFINITION 9. (Element category) Let $\mathcal{C}$ be a category. For a functor $G: \mathcal{C} \rightarrow$ Set, we write $\operatorname{El}(\mathcal{C}, G)$ for the category of pairs of $A \in \mathcal{C}$ and $x \in G A$. Its morphisms are $\mathcal{C}$-morphisms preserving the element. For a functor $G: \mathcal{C}^{\mathrm{op}} \rightarrow$ Set, we define $\operatorname{opEl}(\mathcal{C}, G)=\left(\operatorname{El}\left(\mathcal{C}^{\mathrm{op}}, G\right)\right)^{\mathrm{op}}$. Each has a forgetful functor to $\mathcal{C}$.

DEFINITION 10. (Families construction) Let $\mathcal{C}$ be a category. We write $\operatorname{Fam}(\mathcal{C})$ for the category that has as objects families of objects from $\mathcal{C}$; the homset from $\left(A_{i}\right)_{i \in I}$ to $\left(B_{j}\right)_{j \in J}$ is $\prod_{i \in I} \sum_{j \in J} \mathcal{C}\left(A_{i}, B_{j}\right)$. We write $\operatorname{opFam}(\mathcal{C})$ for $\left(\operatorname{Fam}\left(\mathcal{C}^{\mathrm{op}}\right)\right)^{\mathrm{op}}$. These are respectively the free category with coproducts and the free category with products on $\mathcal{C}$.

\section{DeFINITION 11. Let $\mathcal{C}$ and $\mathcal{D}$ be categories.}

1. A two-dimensional partial map $\mathcal{C} \rightarrow \mathcal{D}$ is a functor $F: \mathcal{C} \rightarrow$ $\operatorname{Fam}(\mathcal{D})$. Equivalently: it consists of a functor $F_{0}: \mathcal{C} \rightarrow$ Set and a functor $F_{1}: \operatorname{El}\left(\mathcal{C}, F_{0}\right) \rightarrow \mathcal{D}$. Any functor $H: \mathcal{D} \rightarrow$ Set gives a functor $\sum_{F} H: \mathcal{C} \rightarrow$ Set defined to be the composite of $H$ with the coproduct-preserving extension of $H$ to $\operatorname{Fam}(\mathcal{D})$. Equivalently: the left Kan extension of $H F^{1}$ along the forgetful functor $\mathrm{El}\left(\mathcal{C}, F_{0}\right) \rightarrow \mathcal{C}$.

2. A 2-dimensional op-partial map $\mathcal{C} \rightarrow \mathcal{D}$ is a functor $F: \mathcal{C} \rightarrow$ $\operatorname{opFam}(\mathcal{D})$. Equivalently: it consists of a functor $F_{0}: \mathcal{C}^{\mathrm{op}} \rightarrow$ Set and a functor $F_{1}: \operatorname{opEl}\left(\mathcal{C}, F_{0}\right) \rightarrow \mathcal{D}$. Any functor $H: \mathcal{D} \rightarrow$ Set gives a functor $\prod_{F} H: \mathcal{C} \rightarrow$ Set defined to be the composite of $H$ with the product-preserving extension of $H$ to $\operatorname{opFam}(\mathcal{D})$. Equivalently: the right Kan extension of $H F^{1}$ along the forgetful functor $\operatorname{opEl}\left(\mathcal{C}, F_{0}\right) \rightarrow \mathcal{C}$.

Now we reformulate our key definitions.

Proposition 6. A categorical game $\mathcal{G}$ consists of

- (small) categories $\mathcal{G}^{\text {pass }}$ and $\mathcal{G}^{\text {act }}$

- a two-dimensional op-partial map Omove $: \mathcal{G}^{\text {pass }} \rightarrow \mathcal{G}^{\text {act }}$

- a two-dimensional partial map Pmove : $\mathcal{G}^{\text {act }} \rightarrow \mathcal{G}^{\text {pass }}$.

PROPOSITION 7. Let $\mathcal{G}$ be a categorical game. 
1. A small-step system over $\mathcal{G}$ is a coalgebra for the endofunctor on $\mathbf{S e t}^{\mathcal{G}^{\text {pass }}} \times$ Set $^{\mathcal{G}^{\text {act }}}$ sending

$$
\left(\mathbb{S}^{\text {pass }}, \mathbb{S}^{\text {act }}\right) \mapsto\left(\prod_{\text {Omove }} \mathbb{S}^{\text {act }},\left(\sum_{\text {Pmove }} \mathbb{S}^{\text {pass }}\right)+\mathbb{S}^{\text {act }}\right)
$$

2. A big-step system over $\mathcal{G}$ is a coalgebra for the endofunctor on Set $^{\mathcal{G}^{\text {pass }}} \times$ Set $^{\mathcal{G}^{\text {act }}}$ sending

$$
\left(\mathbb{S}^{\text {pass }}, \mathbb{S}^{\text {act }}\right) \mapsto\left(\prod_{\text {Omove }} \mathbb{S}^{\text {act }},\left(\sum_{\text {Pmove }} \mathbb{S}^{\text {pass }}\right)+1\right)
$$

3. A passive system over $\mathcal{G}$ is a coalgebra for the endofunctor on Set $^{\mathcal{G}^{\text {pass }}}$ sending

$$
\mathbb{S}^{\text {pass }} \mapsto \prod_{\text {Omove }}\left(\left(\sum_{\text {Pmove }} \mathbb{S}^{\text {pass }}\right)+1\right)
$$

More surprisingly, a game is a coalgebra too:

PRoposition 8. A categorical game is a coalgebra for the endofunctor on Cat $^{2}$ sending

$$
\left(\mathcal{G}^{\text {pass }}, \mathcal{G}^{\text {act }}\right) \mapsto\left(\operatorname{opFam}\left(\mathcal{G}^{\text {act }}\right), \operatorname{Fam}\left(\mathcal{G}^{\text {pass }}\right)\right)
$$

\section{Tensoring}

\subsection{Tensor games}

We often want to run two games $\mathcal{G}$ and $\mathcal{G}^{\prime}$ in parallel, and we describe this by a "tensor" game $\mathcal{G} \otimes \mathcal{G}^{\prime}$, following e.g. [2, 11]. A passive position in the tensor consists of a passive position from each game. O can move in either component; then that component becomes active and $\mathrm{P}$ can only respond within it, whereupon both components are again passive.

DEFINITION 12. For games $\mathcal{G}$ and $\mathcal{G}^{\prime}$, let $\mathcal{G} \otimes \mathcal{G}^{\prime}$ be the following game:

- A passive position is $\left(P, P^{\prime}\right)$ where $P$ and $P^{\prime}$ are passive in $\mathcal{G}$ and $\mathcal{G}^{\prime}$ respectively.

- An active position is either $\operatorname{inl}\left(Q, P^{\prime}\right)$ with $Q$ active in $\mathcal{G}$ and $P^{\prime}$ passive in $\mathcal{G}^{\prime}$, or $\operatorname{inr}\left(P, Q^{\prime}\right)$ with $P$ passive in $\mathcal{G}$ and $Q^{\prime}$ active in $\mathcal{G}^{\prime}$.

- An O-move from $\left(P, P^{\prime}\right)$ is either inl $m$ with $m \in$ Omove $P$, which has target inl $\left(P . m, P^{\prime}\right)$, or inr $m$ with $m \in$ Omove $P^{\prime}$, which has target $\operatorname{inr}\left(P, P^{\prime} . m\right)$.

- A P-move from $\operatorname{inl}\left(Q, P^{\prime}\right)$ is $n \in \operatorname{Pmove} Q$, and its target is $\left(Q . n, P^{\prime}\right)$. Likewise from an inr active position.

DEFINITION 13. For categorical games $\mathcal{G}$ and $\mathcal{G}^{\prime}$, define the categorical game $\mathcal{G} \otimes \mathcal{G}^{\prime}$ as in Definition 12, with the following additional data.

- A passive position morphism from $\left(P, P^{\prime}\right)$ to $\left(P^{\prime \prime}, P^{\prime \prime \prime}\right)$ is a pair $\left(f, f^{\prime}\right)$ with $f: P \rightarrow P^{\prime \prime}$ and $f^{\prime}: P^{\prime} \rightarrow P^{\prime \prime \prime}$. Composite and identity morphisms are defined componentwise.

- Likewise for inl active positions, and likewise for inr active positions. There are no morphisms from inl to inr active positions or vice versa.

\subsection{Tensor strategies}

Any play in $\mathcal{G} \otimes \mathcal{G}^{\prime}$ has a left play and a right play. This is illustrated in Fig. 1.

Proposition 9. Let $\mathcal{G}$ and $\mathcal{G}^{\prime}$ be games. For passive positions $P, P^{\prime}$ in $\mathcal{G}, \mathcal{G}^{\prime}$ respectively, and $\sigma \in \operatorname{Strat}_{\mathcal{G}}^{\text {pass }} P$ and $\sigma^{\prime} \in$ Strat ${ }_{\mathcal{G}}^{\text {pass }} P^{\prime}$, let $\sigma \otimes \sigma^{\prime}$ be the set of passive-ending plays in $\mathcal{G} \otimes \mathcal{G}^{\prime}$ from $\left(P, P^{\prime}\right)$ whose left play is in $\sigma$ and whose right play is in $\sigma^{\prime}$. Then $\sigma \otimes \sigma^{\prime} \in \operatorname{Strat}_{\mathcal{G} \otimes \mathcal{G}^{\prime}}^{\text {pass }}\left(P, P^{\prime}\right)$. Likewise for inl and for inr active positions.
PROPOSITION 10. (Naturality of $\otimes$ )

Let $\mathcal{G}$ and $\mathcal{G}^{\prime}$ be categorical games. For passive position morphisms $f: P \rightarrow P^{\prime \prime}$ in $\mathcal{G}$ and $f^{\prime}: P^{\prime} \rightarrow P^{\prime \prime \prime}$ in $\mathcal{G}^{\prime}$, and $\sigma \in \operatorname{Strat}_{\mathcal{G}}^{\text {pass }} P$ and $\sigma^{\prime} \in$ Strat $_{\mathcal{G}^{\prime}}^{\text {pass }} P^{\prime}$, we have

$$
\begin{aligned}
& \left(\operatorname{Strat}_{\mathcal{G} \otimes \mathcal{G}^{\prime}}^{\text {pass }}\left(f, f^{\prime}\right)\right)\left(\sigma \otimes \sigma^{\prime}\right)= \\
& \quad\left(\operatorname{Strat}_{\mathcal{G}}^{\text {pass }} f\right)(\sigma) \otimes\left(\text { Strat }_{\mathcal{G}^{\prime}}^{\text {pass }} f^{\prime}\right)\left(\sigma^{\prime}\right)
\end{aligned}
$$

Likewise for inl and for inr active positions.

\subsection{Tensor systems}

DEFINITION 14. Let $\mathbb{S}$ and $\mathbb{S}^{\prime}$ be small-step systems over games $\mathcal{G}$ and $\mathcal{G}^{\prime}$ respectively. The small-step system $\mathbb{S} \otimes \mathbb{S}^{\prime}$ over $\mathcal{G} \otimes \mathcal{G}^{\prime}$ is as follows.

- A passive state in position $\left(P, P^{\prime}\right)$ is a pair $\left(x, x^{\prime}\right)$ of states $x \in \mathbb{S}^{\text {pass }} P$ and $x^{\prime} \in \mathbb{S}^{\prime \text { pass }} P^{\prime}$.

- An active state in position $\operatorname{inl}\left(Q, P^{\prime}\right)$ is a pair $\left(y, x^{\prime}\right)$ of states $y \in \mathbb{S}^{\text {act }} Q$ and $x^{\prime} \in \mathbb{S}^{\text {pass }} P^{\prime}$, and likewise for inr.

- $\left(x, x^{\prime}\right) @($ inl $m) \stackrel{\text { def }}{=}\left(x @ m, x^{\prime}\right)$, and likewise for inr $m$.

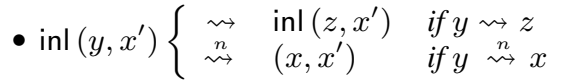

- likewise for $\operatorname{inr}\left(x, y^{\prime}\right)$.

We likewise define the tensor of big-step or passive systems. The following shows that this does not cause ambiguity.

Proposition 11. 1. For small-step systems $\mathbb{S}$ and $\mathbb{S}^{\prime}$ over games $\mathcal{G}$ and $\mathcal{G}^{\prime}$ respectively, the tensor of the big-step forms of $\mathcal{G}$ and $\mathcal{G}^{\prime}$ is the big-step form of $\mathcal{G} \otimes \mathcal{G}^{\prime}$.

2. For big-step systems $\mathbb{S}$ and $\mathbb{S}^{\prime}$ over games $\mathcal{G}$ and $\mathcal{G}^{\prime}$ respectively, the tensor of the passive forms of $\mathcal{G}$ and $\mathcal{G}^{\prime}$ is the passive form of $\mathcal{G} \otimes \mathcal{G}^{\prime}$.

The tensor of systems over categorical games may be defined in the evident way, though we shall not use this.

\subsection{Compositionality theorem for tensors}

We see how to obtain the operational meaning of a tensor state from those of its components.

Proposition 12. Let $\mathcal{G}$ and $\mathcal{G}^{\prime}$ be games.

1. Let $\mathbb{S}$ and $\mathbb{S}^{\prime}$ be passive systems over $\mathcal{G}$ and $\mathcal{G}^{\prime}$ respectively. For $x \in \mathbb{S}^{\text {pass }} P$ and $x^{\prime} \in \mathbb{S}^{\prime \text { pass }} P^{\prime}$ we have

$$
\llbracket\left(x, x^{\prime}\right) \rrbracket_{\left(P, P^{\prime}\right)}^{\text {pass }}=\llbracket x \rrbracket_{P}^{\text {pass }} \otimes \llbracket x^{\prime} \rrbracket_{P^{\prime}}^{\text {pass }}
$$

2. Let $\mathbb{S}$ and $\mathbb{S}^{\prime}$ be big-step systems over $\mathcal{G}$ and $\mathcal{G}^{\prime}$ respectively. For $y \in \mathbb{S}^{\text {act }} Q$ and $x^{\prime} \in \mathbb{S}^{\prime \text { pass }} P^{\prime}$, we have

$$
\llbracket\left(y, x^{\prime}\right) \rrbracket_{\text {inl }\left(Q, P^{\prime}\right)}^{\text {act }}=\llbracket y \rrbracket_{Q}^{\text {act }} \otimes \llbracket x^{\prime} \rrbracket_{P^{\prime}}^{\text {pass }}
$$

Likewise for inr.

By Proposition 11 this result also holds for small-step systems.

\section{Operating on strategies: the example of substitution}

We return to the transition system $\lambda$ Syst for our example calculus. For terms $\mathrm{x}, \mathrm{y} \vdash^{\mathrm{nc}} M$ and $\mathrm{z} \vdash^{\mathrm{v}} V$, suppose we have been given two black boxes: the left one containing active state $\rightarrow M$ in position $\mathrm{x}, \mathrm{y} \|$ and the right one containing passive state $(a \mapsto V)$ in position $\mathbf{z} \| a$. We can play O-moves into them and wait to see what P-moves come out, but cannot see the syntax. We wish to simulate the behaviour of active state $M[V / \mathrm{y}]$, formed by substitution, in position $\mathrm{x}, \mathrm{z} \|$, just using the behaviour from the left and right black boxes. How shall we proceed? We play on three interfaces: with the left and right boxes and with the external world. 
Play in $\mathcal{G} \otimes \mathcal{G}^{\prime} \quad\left(P, P^{\prime}\right) \stackrel{\text { inl } m}{\longrightarrow} \operatorname{inl}\left(P . m, P^{\prime}\right) \stackrel{n}{\longrightarrow}\left(P . m . n, P^{\prime}\right) \stackrel{\text { inr } m^{\prime}}{\longrightarrow} \operatorname{inr}\left(P . m . n, P^{\prime} \cdot m^{\prime}\right) \stackrel{n^{\prime}}{\longrightarrow}\left(P . m . n, P^{\prime} \cdot m^{\prime} . n^{\prime}\right)$

Left play (in $\mathcal{G}) \quad P \odot \quad m \longrightarrow$ P.m $\bullet \quad n$ P.m.n $=$ P.m.n $=$ P.m.n

Right play (in $\mathcal{G}^{\prime}$ ) $\quad P^{\prime}=P^{\prime}=P^{\prime} \odot \quad m^{\prime} \longrightarrow P^{\prime} \cdot m^{\prime} \bullet \quad n^{\prime} \longrightarrow P^{\prime} \cdot m^{\prime} \cdot n^{\prime}$

Figure 1. Left and right play

We first wait for the left box $>M$. If it plays x, then $M \rightsquigarrow^{*}$ $\mathrm{x}\left(W_{0}, W_{1}\right)$ and $\mathrm{O}$ receives fresh names $b_{0}$ and $b_{1}$ representing $W_{0}$ and $W_{1}$ respectively. Thus $M[V / \mathrm{y}] \rightsquigarrow{ }^{*} \mathrm{x}\left(W_{0}[V / \mathrm{y}], W_{1}[V / \mathrm{y}]\right)$ and we make the latter command our current "external state"- the state we are trying to simulate. So we play $\mathrm{x}$ externally, resulting in position $\mathbf{x}, \mathbf{z} \| b_{0}, b_{1}$, and our current external state becomes $\left(b_{0} \mapsto W_{0}[V / y], b_{1} \mapsto W_{1}[V / y]\right)$. We record the fact that $b_{0}$ and $b_{1}$ at the external interface were respectively obtained from $b_{0}$ and $b_{1}$ at the left interface, so that whenever external Opponent plays $b_{0}$, we play $b_{0}$ into the left box.

On the other hand, suppose the left box plays y. Then $M \rightsquigarrow^{*}$ $\mathrm{y}\left(W_{0}, W_{1}\right)$ and $\mathrm{O}$ receives fresh names $b_{0}$ and $b_{1}$ representing $W_{0}$ and $W_{1}$ respectively. Thus $M[V / \mathrm{y}] \rightsquigarrow^{*} V\left(W_{0}[V / \mathrm{y}], W_{1}[V / \mathrm{y}]\right)$ and we make the latter command our current external state. We do not play y externally (indeed the external position is $\mathbf{x}, \mathbf{z} \|$ ). Instead, we play $a$ into the right box, resulting in state $a \mapsto V$ $V\left(\mathrm{w}_{0}, \mathrm{w}_{1}\right)$ in position $\mathbf{z}, \mathrm{w}_{0}, \mathrm{w}_{1} \| a$. We record that $\mathrm{w}_{0}$ and $\mathrm{w}_{1}$ at the right interface were respectively obtained from $b_{0}$ and $b_{1}$ at the the left interface, so that whenever the right box plays wo, we play $b_{0}$ into the left box.

If now the right box plays $\mathbf{z}$, then $V\left(\mathrm{w}_{0}, \mathrm{w}_{1}\right) \rightsquigarrow^{*} \mathbf{z}\left(W_{2}, W_{3}\right)$ so $V\left(W_{0}[V / \mathrm{y}], W_{1}[V / \mathrm{y}]\right) \rightsquigarrow^{*}$

$$
\mathbf{z}\left(W_{2}\left[\begin{array}{l}
W_{0}[V / \mathrm{y}] / \mathrm{w}_{0} \\
W_{1}[V / \mathrm{y}] / \mathrm{w}_{1}
\end{array}\right], W_{3}\left[\begin{array}{l}
W_{0}[V / \mathrm{y}] / \mathrm{w}_{0} \\
W_{1}[V / \mathrm{y}] / \mathrm{w}_{1}
\end{array}\right]\right)
$$

and we make the latter command our current external state. Therefore we play $\mathrm{z}$ at the external interface. Several points may be learnt from this discussion:

1. When we await the external Opponent, all interfaces are passive. When we await a box, it and the external interface are active; the other box is passive.

2. We need to maintain a "linker" function saying where each Oname in the external interface, and each P-name in each internal interface, came from.

3. At any time there is a current external state that we are trying to simulate. It is constructed from chains of substitutions that grow as play continues.

4. When we move between internal (left and right) interfaces, the external state does not change. We must rule out an infinite sequence of consecutive such events, to ensure the external state makes progress during our simulation.

\section{Transfers Between Games}

\subsection{Transfers}

We introduce a notion of transfer from one game to another, which is a recipe for converting a strategy for the first game into a strategy for the second. As we shall see, the procedure outlined in Section 7 forms a transfer $\lambda$ Game $\otimes \lambda$ Game $\rightarrow \lambda$ Game.
Definition 15. Let $\mathcal{G}$ and $\mathcal{H}$ be (discrete) games. $A$ transfer $\mathcal{O}$ : $\mathcal{G} \rightarrow \mathcal{H}$ consists of the following data.

- For each pair of passive positions $P$ in $\mathcal{G}$ and $R$ in $\mathcal{H}$, a set $\mathcal{O}^{\text {pass }}(P, R)$ of passive linkers $P \rightarrow R$.

- For each pair of active positions $Q$ in $\mathcal{G}$ and $S$ in $\mathcal{H}$, a set $\mathcal{O}^{\text {act }}(Q, S)$ of active linkers $Q \rightarrow S$.

- For each passive linker $h: P \rightarrow R$ a function $\gamma_{h}^{\text {pass }} \in$

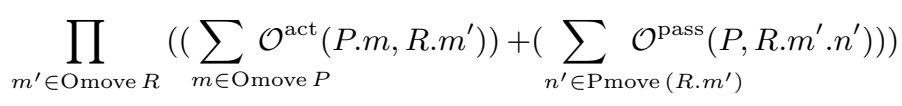

- For each active linker $k: Q \rightarrow S$ a function $\gamma_{k}^{\text {act }} \in$

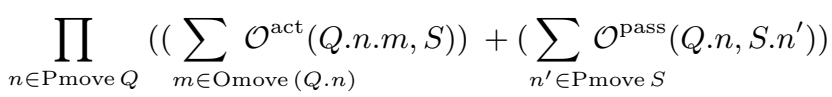

We depict the cases $\gamma_{h}^{\text {pass }}(m)$ of $\operatorname{inl}(m, k)$ and $\operatorname{inr}\left(n^{\prime}, h^{\prime}\right)$ as respectively:

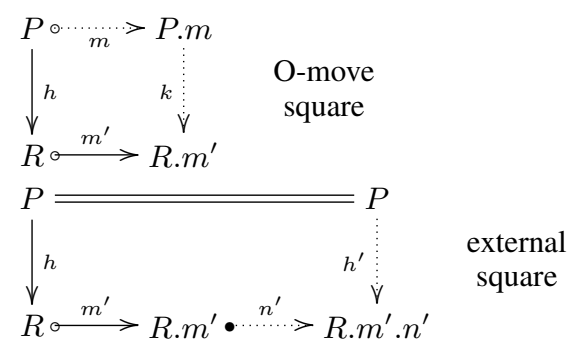

We depict the cases $\gamma_{k}^{\text {act }}\left(n^{\prime}\right)$ of $\operatorname{inl}\left(m, k^{\prime}\right)$ and $\operatorname{inr}\left(n^{\prime}, h\right)$ as respectively:

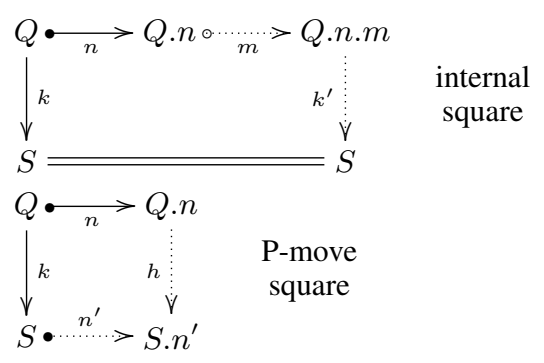

As promised at the start of the section, we give a transfer $\lambda$ Comp from $\lambda$ Game $\otimes \lambda$ Game to $\lambda$ Game. A passive linker $\left(p_{1}, p_{\mathrm{r}}\right) \rightarrow$ $p_{\mathrm{e}}$, where $p_{1}=\Gamma_{1} \| \Delta_{\mathrm{l}}$ etc., is a function $\Gamma_{1}+\Gamma_{\mathrm{r}}+\Delta_{\mathrm{e}} \rightarrow$ $\Delta_{1}+\Delta_{\mathrm{r}}+\Gamma_{\mathrm{e}}$ mapping each name to one at a different interface. (We use inl, inr, ine as ternary sum constructors.) Likewise for active linkers inl $\left(q_{1}, p_{\mathrm{r}}\right) \rightarrow q_{\mathrm{e}}$ or $\operatorname{inr}\left(p_{\mathrm{l}}, q_{\mathrm{r}}\right) \rightarrow q_{\mathrm{e}}$. The squares are 
- if $h($ ine $a)=\operatorname{inl} b$ then O-move square

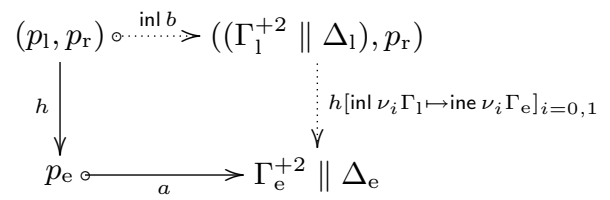

and likewise if $h($ ine $a)=\operatorname{inr} b$

- if $k($ inl $\mathrm{x})=$ ine y then P-move square

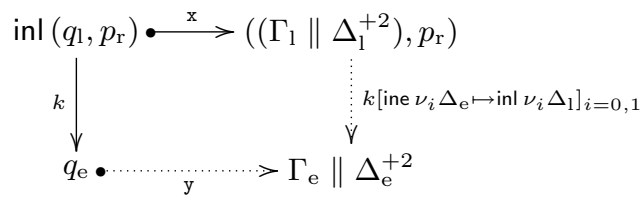

and if $k(\mathrm{inl} \mathrm{x})=\operatorname{inr} a$ then internal square

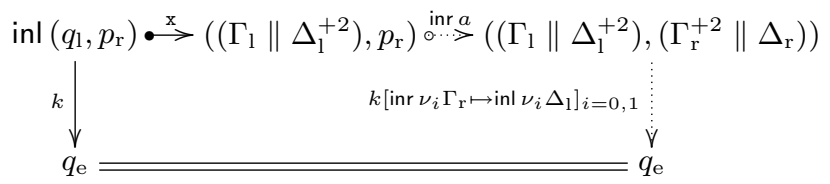

- likewise for inr.

To define a transfer between categorical games, we need the well-known notion of bimodule (also called "profunctor" and "distributor").

Definition 16. Let $\mathcal{C}$ and $\mathcal{D}$ be categories. $A(\mathcal{C}, \mathcal{D})$-bimodule $\mathcal{M}$ consists of the following data:

- for each $X \in \mathcal{C}$ and $Y \in \mathcal{D}$ a set $\mathcal{M}(X, Y)$ of $\mathcal{M}$-morphisms $X \rightarrow Y$

- for any $\mathcal{C}$ - and $\mathcal{M}$-morphisms $X^{\prime} \stackrel{f}{\rightarrow} X \stackrel{g}{\rightarrow} Y$ an $\mathcal{M}$-morphism $X^{\prime} \stackrel{f ; g}{\longrightarrow} Y$

- for any $\mathcal{M}$ - and D-morphisms $X \stackrel{g}{\rightarrow} Y \stackrel{h}{\rightarrow} Y^{\prime}$ an $\mathcal{M}$-morphism $X \stackrel{g ; h}{\longrightarrow} Y^{\prime}$

The following five equations must be satisfied:

- For a M-morphism $g: X \rightarrow Y$ we have $\operatorname{id}_{X} ; g=g=$ $g ; \operatorname{id}_{Y}$.

- For two $\mathcal{C}$-morphisms and an $\mathcal{M}$-morphism:

$$
X^{\prime \prime} \stackrel{f^{\prime}}{\longrightarrow} X^{\prime} \stackrel{f}{\longrightarrow} X \stackrel{g}{\longrightarrow} Y
$$

we have $\left(f^{\prime} ; f\right) ; g=f^{\prime} ;(f ; g)$

- For a $\mathcal{C}$-morphism and $\mathcal{M}$-morphism and $\mathcal{D}$-morphism:

$$
X^{\prime} \stackrel{f}{\longrightarrow} X \stackrel{g}{\longrightarrow} Y \stackrel{h}{\longrightarrow} Y^{\prime}
$$

we have $(f ; g) ; h=f ;(g ; h)$

- For a $\mathcal{M}$-morphism and two D-morphisms

$$
X \stackrel{g}{\longrightarrow} Y \stackrel{h}{\longrightarrow} Y^{\prime} \stackrel{h^{\prime}}{\longrightarrow} Y^{\prime \prime}
$$

we have $(g ; h) ; h^{\prime}=g ;\left(h ; h^{\prime}\right)$.

Concisely: $a(\mathcal{C}, \mathcal{D})$-bimodule is a functor $\mathcal{C}^{\mathrm{op}} \times \mathcal{D} \rightarrow$ Set.

Definition 17. Let $\mathcal{G}$ and $\mathcal{H}$ be categorical games. A transfer $\mathcal{O}: \mathcal{G} \rightarrow \mathcal{H}$ is defined as in Def. 15 , with the following additional data.
- Composition of a passive linker $P \rightarrow R$ with a passive position morphism $P^{\prime} \rightarrow P$ in $\mathcal{G}$, and with a passive position morphism $R \rightarrow R^{\prime}$ in $\mathcal{H}$, satisfying the five equations in Def. 16 .

- Likewise for active linkers.

We require all composites

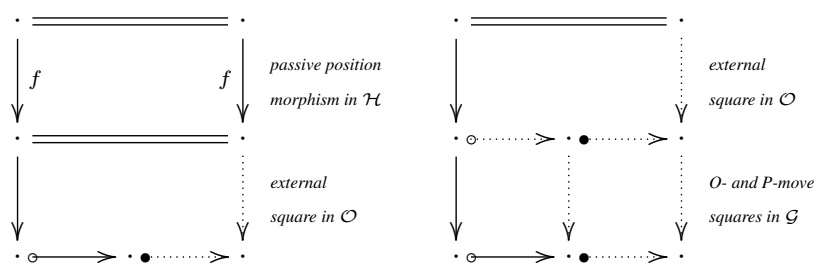

to be external squares, all composites
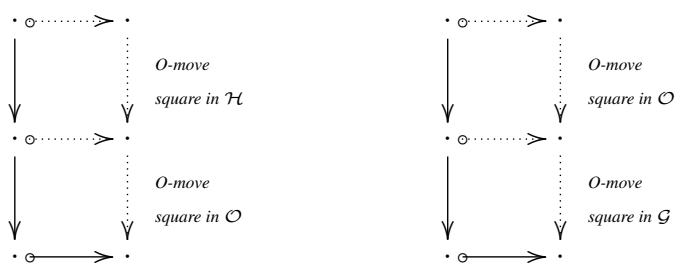

to be O-move squares, and likewise for P-move and internal squares.

Our example $\lambda$ Comp is a categorical transfer: linker composition is given by function composition in the evident way.

\subsection{Transfer operating on strategies}

To define how a transfer $\mathcal{O}$ operates on strategies, we consider "O-interaction sequences" comprised of O-move, external, P-move and internal squares. An example is shown in Fig. 2 We may describe this interaction sequence from $h$ by the sequence of moves $m_{0}^{\prime}, n_{0}, n_{1}, m_{1}^{\prime}, n_{2}, m_{2}^{\prime}$ depicted as solid, since they determine the other moves and morphisms. The play from $P$ appearing along the upper edge is called the internal play; the play from $R$ appearing along the lower edge is called the external play.

Proposition 13. Let $\mathcal{O}: \mathcal{G} \rightarrow \mathcal{H}$ be a transfer. For any passive linker $h: P \rightarrow R$ and strategy $\sigma \in \operatorname{Strat}_{\mathcal{G}}^{\text {pass }} P$ let $\left(\operatorname{Strat}_{\mathcal{O}}^{\text {pass }} h\right) \sigma$ be the set of all external plays of passive-ending $\mathcal{O}$-interaction sequences from $h$ whose internal play is in $\sigma$. Then ( $\left.\operatorname{Strat}_{\mathcal{O}}^{\text {pass }} h\right) \sigma \in$ Strat $_{\mathcal{H}}^{\text {pass }} R$. Likewise for an active linker.

Proposition 14. Let $\mathcal{O}: \mathcal{G} \rightarrow \mathcal{H}$ be a transfer of categorical games.

\section{For passive position morphism in $\mathcal{G}$ and linker}

$$
P^{\prime} \stackrel{f}{\longrightarrow} P \stackrel{h}{\longrightarrow} R
$$

and strategy in Strat $_{\mathcal{G}}^{\text {pass }} R^{\prime}$ we have

$$
\left(\operatorname{Strat}_{\mathcal{O}}^{\text {pass }}(f ; h)\right)(\sigma)=\left(\operatorname{Strat}_{\mathcal{O}}^{\text {pass }} h\right)\left(\operatorname{Strat}_{\mathcal{G}}^{\text {pass }} f\right)(\sigma)
$$

Likewise for active positions.

2. For passive linker and position morphism in $\mathcal{H}$

$$
P \stackrel{h}{\longrightarrow} R \stackrel{f}{\longrightarrow} R^{\prime}
$$

and strategy in $\mathrm{Strat}_{\mathcal{G}}^{\text {pass }} R$ we have

$$
\left(\operatorname{Strat}_{\mathcal{O}}^{\text {pass }}(h ; f)\right)(\sigma)=\left(\operatorname{Strat}_{\mathcal{H}}^{\text {pass }} f\right)\left(\operatorname{Strat}_{\mathcal{O}}^{\text {pass }} h\right)(\sigma)
$$

Likewise for active positions. 


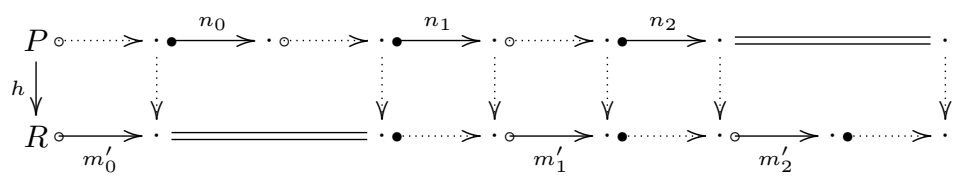

Figure 2. An interaction sequence

\subsection{Stepped bisimulation across a transfer}

We now establish the relationship between transition systems and transfers, i.e. between operational and denotational game semantics. We shall consider only discrete games.

To ensure that we do not perform infinitely many operations at the internal interface without making progress, we "grade" the states to give a finite quota of internal operations.

Definition 18. Given a transfer $\mathcal{O}: \mathcal{G} \rightarrow \mathcal{H}$ and small-step systems $\mathbb{S}$ over $\mathcal{G}$ and $\mathbb{T}$ over $\mathcal{H}$, a stepped bisimulation from $\mathbb{S}$ to $\mathbb{T}$ across $\mathcal{O}$ consists of the following data.

- For each passive linker $h: P \rightarrow R$, a relation $\mathcal{R}_{h}^{\text {pass }}$ from $\mathbb{S}^{\text {pass }} P$ to $\mathbb{T}^{\text {pass }} R$.

- For each active linker $k: Q \rightarrow S$,

- a subset $\mathcal{U}_{k}$ of $\mathbb{S}^{\text {act }} Q$

- an increasing sequence $\left(\mathcal{V}_{k}^{i}\right)_{i \in \mathbb{N}}$ of subsets of $\mathcal{U}_{k}$, such that $\mathcal{U}_{k}=\bigcup_{i \in \mathbb{N}} \mathcal{V}_{k}^{i}$

- and a relation $\mathcal{R}_{k}^{\text {act }}$ from $\mathcal{U}_{k}$ to $\mathbb{T}^{\text {act }} S$.

These are required to satisfy the following conditions.

- For any passive linker $h: P \rightarrow R$ and $x\left(\mathcal{R}_{h}^{\text {pass }}\right) x^{\prime}$ and $m^{\prime} \in$ Omove $R$,

- if $m$ completes to an $O$-move square

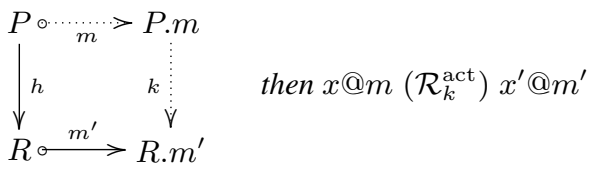

- if $m$ completes to an external square

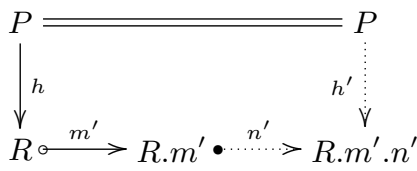

then $x^{\prime} @ m^{\prime} \stackrel{n}{\rightsquigarrow} w^{\prime}$ with $x\left(\mathcal{R}_{h^{\prime}}^{\text {pass }}\right) w^{\prime}$.

- For any active linker $k: Q \rightarrow S$ and $i \in \mathbb{N}$ and $y \in \mathcal{V}_{k}^{i}$, if $y \stackrel{n}{\rightsquigarrow} x$ and $n$ completes to an internal square

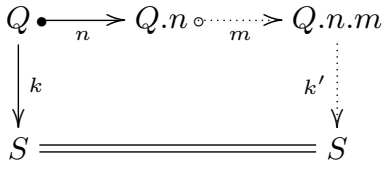

then $x @ m \in \mathcal{V}_{k^{\prime}}^{j}$ for some $j<i$.

- For any active linker $k: Q \rightarrow S$ and $y\left(\mathcal{R}_{k}^{\text {act }}\right) y^{\prime}$,

- if $y \rightsquigarrow z$ then $y^{\prime} \rightsquigarrow z^{\prime}$ with $z\left(\mathcal{R}_{k}^{\text {act }}\right) z^{\prime}$

- if $y \stackrel{n}{\sim} x$ and $n$ completes to a P-move square

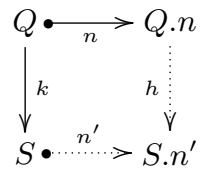

then $y^{\prime} \stackrel{n^{\prime}}{\rightsquigarrow} x^{\prime}$ with $x\left(\mathcal{R}_{h}^{\text {pass }}\right) x^{\prime}$

- if $y \stackrel{n}{\rightsquigarrow} x$ and $n$ completes to an internal square (4) then $x @ m\left(\mathcal{R}_{k^{\prime}}^{\mathrm{act}}\right) y^{\prime}$.

Recall our example transfer $\lambda$ Comp $: \lambda$ Game $\otimes \lambda$ Game $\rightarrow$ $\lambda$ Game. We construct a stepped bisimulation $\left(\mathcal{R}^{\text {pass }}, \mathcal{U}, \mathcal{V}, \mathcal{R}^{\text {act }}\right)$ from $\lambda$ Syst $\otimes \lambda$ Syst to $\lambda$ Syst across this transfer.

Given a passive linker $h:\left(p_{1}, p_{\mathrm{r}}\right) \rightarrow p_{\mathrm{e}}$, an $h$-grading $\phi$ consists of a natural number $|\phi|$ (the number of "generations") and maps $\phi_{1}: \Delta_{1} \rightarrow \$|\phi|$ and $\phi_{\mathrm{r}}: \Delta_{\mathrm{r}} \rightarrow \$|\phi|$ (saying when each name was generated). For an $h$-grading $\phi$ and $i \in \mathbb{N}$ we write Older $_{1}^{\phi} i \subseteq \Gamma_{1}$ and Older ${ }_{\mathrm{r}}^{\phi} i \subseteq \Gamma_{\mathrm{r}}$ for the names that are older than generation $i$. Explicitly: Older ${ }_{1}^{\phi} i$ consists of those $\mathrm{x} \in \Gamma_{1}$ such that $k($ inl $\mathrm{x})$ is either inr $a$ with $\phi_{\mathrm{r}}(a)<i$ or ine $\mathrm{y}$. (External P-names are deemed to have always existed.)

Now a passive state in position $\left(p_{1}, p_{\mathrm{r}}\right)$ takes the form

$$
\left(\left(V_{a}^{\mathrm{l}}\right)_{a \in \Delta_{1}},\left(V_{a}^{\mathrm{r}}\right)_{a \in \Delta_{\mathrm{r}}}\right)
$$

The state (5) is graded by an $h$-grading $\phi$ when for all $a \in \Delta_{\mathrm{l}}$ we have Older ${ }_{1}^{\phi} \phi_{1}(a) \vdash^{\vee} V_{a}^{1}$ and likewise for $a \in \Delta_{\mathrm{r}}$. If $[5]$ is $h$ gradeable (i.e. is graded by some $h$-grading), then we shall define its $h$-substitution, a passive state in position $p_{\mathrm{e}}$ as follows. First associate to each $u \in \Delta_{\mathrm{l}}+\Delta_{\mathrm{r}}+\Gamma_{\mathrm{e}}$ a value $\Gamma_{\mathrm{e}} \vdash^{\vee} W_{u}$ by the equations

$$
\begin{aligned}
W_{\text {inl } a} & =V_{a}^{1}\left[W_{h(\text { inl } \mathrm{x})} / \mathrm{x}\right]_{\mathrm{x} \in \Gamma_{1}} \\
W_{\text {inr } a} & =V_{a}^{\mathrm{r}}\left[W_{h(\text { inr } \mathrm{x})} / \mathrm{x}\right]_{\mathrm{x} \in \Gamma_{\mathrm{r}}} \\
W_{\text {ine } \mathrm{x}} & =\mathrm{x}
\end{aligned}
$$

which have a unique solution by induction over a grading. Then define the $h$-substitution of 5 to be $\left(W_{h \text { (ine } a)}\right)_{a \in \Delta_{\mathrm{e}}}$.

We set $\mathcal{R}_{h}^{\text {pass }}$ to be the partial function relating each $h$-gradeable state (5) to its $h$-substitution.

Given an active linker $k: \operatorname{inl}\left(q_{1}, p_{\mathrm{r}}\right) \rightarrow q_{\mathrm{e}}$ we likewise define a $k$-grading. An active state in position inl $\left(q_{1}, p_{\mathrm{r}}\right)$ takes the form

$$
\left(\left(V_{a}^{\mathrm{l}}\right)_{a \in \Delta_{1}} \triangleright M,\left(V_{a}^{\mathrm{r}}\right)_{a \in \Delta_{\mathrm{r}}}\right)
$$

The state (6) is graded by a $k$-grading $\phi$ when for all $a \in \Delta_{1}$ we have $\operatorname{Older}_{1}^{\phi} \phi_{1}(a) \vdash^{v} V_{a}^{1}$ and likewise for $a \in \Delta_{\mathrm{r}}$. For $i \in \mathbb{N}$, the state (6) is graded at level $i$ by $\phi$ when the following additional condition holds:

$$
M=V\left(W, W^{\prime}\right) \quad \text { implies } \quad \operatorname{Older}_{1}^{\phi} i \vdash^{\vee} V .
$$

If 6 is $k$-gradeable, we define its $k$-substitution, an active state in position $q_{\mathrm{e}}$, to be

$$
\left(W_{k(\text { ine } a)}\right)_{a \in \Delta_{\mathrm{e}}}>M\left[W_{k(\text { inl } \mathrm{x})} / \mathrm{x}\right]_{\mathrm{x} \in \Gamma_{1}}
$$

where $W_{u}$ is defined as in the passive case.

We set $\mathcal{U}_{k}$ to be the set of active states (6) that are $k$-gradeable, and $\mathcal{V}_{k}^{i}$ to be the set of those that are $k$-gradeable at level $i$. We set $\mathcal{R}_{k}^{\text {act }}$ to be the function relating each $k$-gradeable state (6) to its $k$-substitution. Likewise for the inr case.

It is then straightforward to verify that $\left(\mathcal{R}^{\text {pass }}, \mathcal{U}, \mathcal{V}, \mathcal{R}^{\text {act }}\right)$ is a stepped bisimulation across $\lambda$ Comp. 


\subsection{Compositionality theorem for transfers}

Proposition 15. Given a transfer $\mathcal{O}: \mathcal{G} \rightarrow \mathcal{H}$ and smallstep systems $\mathbb{S}$ over $\mathcal{G}$ and $\mathbb{T}$ over $\mathcal{H}$, let $\left(\mathcal{R}^{\text {pass }}, \mathcal{U}, \mathcal{V}, \mathcal{R}^{\text {act }}\right)$ be a stepped bisimulation from $\mathbb{S}$ to $\mathbb{T}$ across $\mathcal{O}$.

1. For a passive linker $h: P \rightarrow R$, if $x\left(\mathcal{R}_{h}^{\text {pass }}\right) x^{\prime}$ then

$$
\llbracket x^{\prime} \rrbracket_{R}^{\text {pass }}=\left(\text { Strat }_{\mathcal{O}}^{\text {pass }} h\right) \llbracket x \rrbracket_{P}^{\text {pass }}
$$

2. For an active linker $k: Q \rightarrow S$, if $y\left(\mathcal{R}_{k}^{\text {act }}\right) y^{\prime}$ then

$$
\llbracket y^{\prime} \rrbracket_{S}^{\text {pass }}=\left(\operatorname{Strat}_{\mathcal{O}}^{\text {act }} k\right) \llbracket y \rrbracket_{Q}^{\text {act }}
$$

We illustrate this with our example. Let the active linker

$$
k: \operatorname{inl}((\mathrm{x}, \mathrm{y} \|),(\mathbf{z} \| a)) \rightarrow(\mathrm{x}, \mathbf{z} \|)
$$

send inl $\mathrm{x} \mapsto$ ine $\mathrm{x}$ and inl $\mathrm{y} \mapsto \operatorname{inr} a$ and inr $\mathrm{z} \mapsto$ ine $\mathrm{z}$. The state $\operatorname{inl}(\triangleright M,(a \mapsto V))$ is graded by the $k$-grading $(1,(),(a \mapsto 0))$ and its $k$-substitution is $M[V / y]$. Since $\left(\mathcal{R}^{\text {pass }}, \mathcal{U}, \mathcal{V}, \mathcal{R}^{\text {act }}\right)$ is a stepped bisimulation across $\lambda$ Comp, we obtain

$$
\begin{aligned}
& \llbracket \rightarrow M[V / \mathrm{y}] \rrbracket_{\mathrm{x}, \mathrm{z} \|}^{\mathrm{act}} \\
& =\left(\operatorname{Strat}_{\lambda \text { Comp }}^{\text {act }} k\right) \llbracket \operatorname{inl}(\longrightarrow M,(a \mapsto V)) \rrbracket_{\text {inl }}^{\text {act }}((\mathrm{x}, \mathrm{y} \|),(\mathrm{z} \| a)) \\
& \text { (by Prop. 15. } \\
& =\left(\operatorname{Strat}_{\lambda \mathbf{C o m p}}^{\text {act }} k\right)\left(\llbracket \triangleright M \rrbracket_{\mathrm{x}, \mathrm{y} \|}^{\mathrm{act}} \otimes \llbracket a \mapsto V \rrbracket_{\mathrm{z} \| a}^{\mathrm{pass}}\right) \\
& \text { (by Prop. 12) }
\end{aligned}
$$

This validates the procedure described in Sect.7.

\section{Dual Games and Transfers}

There is an evident involution on games:

DEFINITION 19. For a categorical game

$$
\mathcal{G}=\left(\mathcal{G}^{\text {pass }}, \mathcal{G}^{\text {act }}, \text { Omove }, \text { Pmove }\right)
$$

its dual is given by

$$
\mathcal{G}^{\perp} \stackrel{\text { def }}{=}\left(\left(\mathcal{G}^{\text {act }}\right)^{\text {op }},\left(\mathcal{G}^{\text {pass }}\right)^{\text {op }}, \text { Pmove }{ }^{\text {op }}, \text { Omove }{ }^{\text {op }}\right)
$$

This provides a concise formulation of transfer:

Proposition 16. Let $\mathcal{G}$ and $\mathcal{H}$ be categorical games. A transfer $\mathcal{G} \rightarrow \mathcal{H}$ is a total passive system over $\mathcal{G} \multimap \mathcal{H} \stackrel{\text { def }}{=}\left(\mathcal{G} \otimes \mathcal{H}^{\perp}\right)^{\perp}$

Remark Transfers cannot be composed, because of the possibility of "infinite chattering" [2], but partial transfers can be, giving rise to a $*$-autonomous bicategory.

\section{Conclusions and Future Work}

We have described a basic framework with a "checklist" for formulating a game model of a language:

- give a small-step system over a categorical game

- for each syntactic operation, give a transfer, and a stepped bisimulation across it to demonstrate its correctness.

It remains to examine the many game models in the literature that use justification pointers to see how well they fit this framework. We shall need to study transfers that create multiple threads of the strategy they act on, as in [11]. We also should treat nondeterministic systems, where there is a proliferation of notions of equivalence, hence of strategy. A final intriguing question is whether our sequential framework can be adapted for concurrent systems.

\section{References}

[1] Abramsky, S.: The lazy $\lambda$-calculus. In: Research topics in Functional Programming, pp. 65-117. Addison Wesley (1990)

[2] Abramsky, S.: Semantics of interaction: an introduction to game semantics. In: 1996 CLiCS Summer School. Cambridge U. Press (1997)

[3] Abramsky, S., Honda, K., McCusker, G.: A fully abstract game semantics for general references. In: 13th LICS (1998)

[4] Blum, W., Ong, C.H.L.: The safe lambda calculus. Logical Methods in Computer Science 5(1) (2009)

[5] Curien, P.L.: Abstract Böhm trees. Math. Structures in CS 8(6) (1998)

[6] Danos, V., Herbelin, H., Regnier, L.: Game semantics and abstract machines. In: 11th LICS (1996)

[7] Ghica, D.R., Tzevelekos, N.: A system-level game semantics. 28th Mathematical Foundations in Programming Semantics, ENTCS 286

[8] Harmer, R., Hyland, M., Melliès, P-A.: Categorical combinatorics for innocent strategies. LICS 2007: 379-388

[9] Howe, D.J.: Proving congruence of bisimulation in functional programming languages. Information and Computation 124(2) (1996)

[10] Hyland, J.M.E., Ong, C.H.L.: On full abstraction for PCF: I, II, and III. Information and Computation 163(2) (2000)

[11] Hyland, J.M.E.: Game semantics. In: Semantics and Logics of Computation. Cambridge University Press (1997)

[12] Hyvernat, P.: A linear category of polynomial diagrams. Mathematical Structures in Computer Science 24 (2013)

[13] Jagadeesan, R., Pitcher, C.S., Riely, J.: Open bisimulation for aspects. In: Aspect-Oriented System Development. ACM Press (2007)

[14] Jagadeesan, R., Pitcher, C., Rathke, J., Riely, J.: Local memory via layout randomization. In: 24th Computer Security Foundations Symposium (2011)

[15] Jeffrey, A., Rathke, J.: Java Jr.: Fully abstract trace semantics for a core Java language. European Symp. on Programming, LNCS 3444 (2005)

[16] Jeffrey, A., Rathke, J.: A fully abstract may testing semantics for concurrent objects. Theoretical Computer Science 338 (2005)

[17] Kozen, D.: Realization of coinductive types. In: 27th Conference on the Math. Foundations of Programming Semantics ENTCS 276 (2011)

[18] Lack, S., Street, R.: The formal theory of monads II. Journal of Pure and Applied Algebra 175 (2002)

[19] Laird, J.: Full abstraction for functional languages with control. In: 12th LICS (1997)

[20] Laird, J.: A fully abstract game semantics of local exceptions. In: 16th LICS (2001)

[21] Laird, J.: A fully abstract trace semantics for general references. In 34th ICALP, LNCS 4596 (2007)

[22] Laird, J.: Game semantics for a polymorphic programming language. In: LICS (2010)

[23] Laird, J.: Game semantics for call-by-value polymorphism. In: 37th ICALP, LNCS 6199 (2010)

[24] Lassen, S.B.: Eager normal form bisimulation. In: 20th LICS (2005)

[25] Lassen, S.B., Levy, P.B.: Typed normal form bisimulation. In: 23rd CSL, LNCS, 4646 (2007)

[26] Lassen, S.B., Levy, P.B.: Typed normal form bisimulation for parametric polymorphism. In: LICS (2008)

[27] Murawski, A.S., Tzevelekos, N.: Game semantics for good general references. In: LICS (2011)

[28] Sangiorgi, D.: The lazy lambda calculus in a concurrency scenario. Information and Computation 111(1), 120-153 (1994)

[29] Støvring, K., Lassen, S.: A complete, co-inductive syntactic theory of sequential control and state. In: Semantics and Algebraic Specification, P. Mosses 60th birthday festschrift, LNCS 5700 (2009)

[30] Turi, Plotkin: Towards a mathematical operational semantics. In: LICS: IEEE Symposium on Logic in Computer Science (1997)

[31] Winskel, G: Strategies as Profunctors. In: FoSSaCS 2013. 